\title{
A G-Rich Sequence within the c-kit Oncogene Promoter Forms a Parallel G-Quadruplex Having Asymmetric G-Tetrad Dynamics
}

\author{
Shang-Te Danny Hsu ${ }^{\dagger}$, Peter Varnai ${ }^{\ddagger}$, Anthony Bugaut ${ }^{\dagger}$, Anthony P. Reszka ${ }^{\S}$, Stephen \\ Neidle $^{\star}, \S$, and Shankar Balasubramanian ${ }^{\star}, \dagger, \perp$ \\ † University Chemical Laboratory, University of Cambridge, Lensfield Road, Cambridge, CB2 \\ $1 \mathrm{EW}$, United Kingdom \\ ‡ Department of Chemistry and Biochemistry, University of Sussex, Falmer Brighton BN1 9QJ, \\ United Kingdom \\ $\S$ The Cancer Research UK, Biomolecular Structure Group, The School of Pharmacy, University \\ of London, 29-39 Brunswick Square, London WC1N 1AX, United Kingdom
}

${ }^{\perp}$ School of Clinical Medicine, University of Cambridge, Cambridge CB2 0SP, United Kingdom

\begin{abstract}
Guanine-rich DNA sequences with the ability to form quadruplex structures are enriched in the promoter regions of protein-coding genes, particularly those of proto-oncogenes. G-quadruplexes are structurally polymorphic and their folding topologies can depend on the sample conditions. We report here on a structural study using solution state NMR spectroscopy of a second Gquadruplex-forming motif (c-kit2) that has been recently identified in the promoter region of the $c$ kit oncogene. In the presence of potassium ions, $c$-kit2 exists as an ensemble of structures that share the same parallel-stranded propeller-type conformations. Subtle differences in structural dynamics have been identified using hydrogen-deuterium exchange experiments by NMR spectroscopy, suggesting the coexistence of at least two structurally similar but dynamically distinct substates, which undergo slow interconversion on the NMR timescale.
\end{abstract}

\section{Introduction}

DNA and RNA sequences containing repeated short guanine-rich tracts can form higherorder structures termed G-quadruplexes in which the underlying motif is four in-plane guanine bases hydrogen-bonded together to form a G-tetrad. ${ }^{1}$ G-quadruplex arrangements can be tetramolecular, bimolecular, or intramolecular. A large number of quadruplex structures are possible by virtue of the variation in strand polarities and also the sequence and topologies of the loops (for bimolecular or intramolecular quadruplexes). Intramolecular G-quadruplexes typically comprise several G-tetrads stacked one on top of each other with intervening looped-out sequences. ${ }^{2}{ }^{3}$ The human telomeric intramolecular DNA Gquadruplex is the best studied quadruplex system and was first characterized in $\mathrm{Na}^{+}$solution by NMR to reveal an antiparallel topology with one diagonal and two lateral loops. ${ }^{4}$ The crystal structure of the same system in $\mathrm{K}^{+}$showed an all-parallel topology with three chainreversal loops. ${ }^{5}$ More recently, studies have shown that the telomeric sequence can form a

(C) 2009 American Chemical Society

stephen.neidle@pharmacy.ac.uk; sb10031@cam.ac.uk.

Supporting Information Available: Additional 1D and 2D NOESY spectra of c-kit2 variants recorded under different conditions. Imino proton chemical shift assignment of $c$-kit2 loop variants. Complete authorship for ref 79 . This material is available free of charge via the Internet at http://pubs.acs.org. 
mixed $(3+1)$ structure comprising a single chain-reversal loop and two lateral loops. ${ }^{6}$ Such studies have shown that G-quadruplex topologies can be sensitive to the experimental conditions. Quadruplex nucleic acid sequences have been located in human and other genomes, ${ }^{7}, 8$ with noteworthy overrepresentation in promoter sequences of genes involved in cellular proliferation. ${ }^{9}{ }^{11}$ As yet, the topology and molecular structures have been characterized for only a few oncogene promoter sequences that form G-quadruplex structures. ${ }^{12}{ }^{14}$ In contrast with the human telomeric G-quadruplex which has three trinucleotide loops, promoter G-quadruplexes appear to generally have one or more short loops that are predisposed to forming chain-reversal loops and promoting parallel quadruplex topology. ${ }^{15},{ }^{16}$ The $c$-myc oncogene has been studied in detail, ${ }^{17},{ }^{18}$ as the paradigm for promoter G-quadruplexes, with solution structures being reported for several G-quadruplexes using nuclear magnetic resonance (NMR) spectroscopy, ${ }^{19},{ }^{20}$ from within the NHE III 1 element of the $c$-myc promoter. It has been suggested that selective targeting of a promoter oncogene quadruplex by a small molecule may stabilize its tertiary structure, and that this may be of therapeutic utility. ${ }^{17},{ }^{21}$ Down-regulation of $c$-myc expression has been demonstrated using the quadruplex-binding porphyrin molecule TMPyP4 ${ }^{18}$ and the quindoline derivative SYUIQ-5, ${ }^{22}$ and the supercoiling implications of G-quadruplex formation within a duplex DNA environment have been recently explored. ${ }^{23}$ The Gquadruplex promoter targeting approach offers an alternative to the direct drug targeting of oncogenic kinase protein active sites, which has often resulted in severe clinical problems of resistance to these drugs, usually via active-site mutations.

The proto-oncogene $c$-kit codes for a 145-160 kDa tyrosine kinase receptor, which regulates several important signal transduction cascades that control cell growth and proliferation. ${ }^{24}{ }^{26}$ Functionally significant mutations and overexpression of the $c$-kit protein play a role in oncogenic transformation of certain cell types and are found in several highly malignant human cancers, notably gastrointestinal stromal tumors (GIST), in which a majority of GIST cells contain activating mutations in $c$-kit. ${ }^{27}$ The drug imatinib has recently been licensed for clinical use in the treatment of GIST; its mode of action involves binding in the ATP pocket of the $c$-kit kinase domain and maintaining it in an inactive conformation. ${ }^{28}{ }_{-30}$ This directly results in significant tumor regression, but resistance to the drug eventually occurs in almost all patients. ${ }^{31}, 32$ Newer kinase inhibitors have been developed, such as the compound sunitinib, ${ }^{33}, 34$ however, the heterogeneity of kinase mutations and of resistance mechanisms suggests that the clinical challenges of resistance still remain to be overcome. ${ }^{35} \mathrm{We}$ have used small molecules to target G-quadruplex DNA structures in the c-kitgene, ${ }^{36}$ and have recently shown that cell growth can be halted in a panel of clinically derived GIST cell lines accompanied by a $>90 \%$ decrease in $c$-kit expression. ${ }^{37}$ Detailed structural descriptions of the G-quadruplexes in the $c$-kit gene will therefore be of great interest for structure-based small molecule developments.

We have previously identified two sequences (c-kit1 and c-kit2) that occur in a nuclease hypersensitive region of the promoter ${ }^{38}$ of the $c$-kit oncogene, upstream of the transcription start site, and have shown them as being capable of forming G-quadruplex structures. ${ }^{39}, 40$ The three-dimensional (3D) structure of the c-kit $1 \mathrm{G}$-quadruplex has been determined by solution state NMR spectroscopy and shown to form a unique topology. ${ }^{41},{ }^{42}$ We report here on the topology and structure of the c-kit2 quadruplex, whose sequence we have previously shown to promote the formation of nonduplex structure within the context of a natural DNA duplex sequence. ${ }^{43}$ The $c$-kit2 G-quadruplex adopts an all-parallel propeller-like topology with clear evidence of structural polymorphism, manifested by the presence of typically two sets of resonances in the imino proton NMR spectra, corresponding to regions of the tetrads that are asymmetrically distributed. The same asymmetry has been characterized in terms of local dynamics through hydrogen-deuterium exchange (HDX) experiments based on which 
the presence of at least two dynamically distinct (but structurally very similar) populations are identified.

\section{Results}

\section{c-kit2 Adopts a Parallel G-Quadruplex Topology with a Long Loop}

We have previously employed solution state NMR spectroscopy to show that the 21nucleotide DNA sequence from $c-k i t 2,5^{\prime}-\mathrm{CG}_{3} \mathrm{CG}_{3} \mathrm{CGCGAG}_{3} \mathrm{AG}_{4}-3^{\prime}$, contains highly protected imino protons that we attributed as evidence for G-quadruplex structure formation. 40 The large number (i.e., >12) of observed imino proton resonances in the wild-type (wt) sequence however, suggests that the $c$-kit2 quadruplex is structurally heterogeneous due to the four consecutive guanines at the $3^{\prime}$-end, each of which could in principle participate in G-tetrad formation. Indeed, a single nucleotide replacement at the last position, designated as G21T, gave rise to a much more simplified imino proton NMR spectrum, indicative of fewer G-quadruplex structures; the total number of observed imino proton resonances remained in excess of the 12 that would have been expected from three G-tetrads (four imino protons per tetrad) of a single structure. The observed structural heterogeneity appeared to be kinetically driven and was insensitive to the amount of $\mathrm{K}^{+}$ions present (from 10 to $100 \mathrm{mM}$ ) and DNA concentrations (from $100 \mu \mathrm{M}$ to $1 \mathrm{mM}$ ). After a prolonged incubation time, the imino proton NMR spectrum of the wt $c$-kit2 sample eventually shows 12 expected imino proton resonances, as does the G21T sample, while the time required for the latter to reach apparent homogeneity is much shorter (see Figure S1 in Supporting Information, SI). Despite the structural heterogeneity, the unimolecular conformations in wt c-kit2 and G21T are very similar as shown by the superimposable crosspeaks in the nuclear Overhauser effect (NOE) spectra (Figure S2). There were minor differences in the experimental conditions used here compared to our previous study, ${ }^{40}$ which include the DNA concentration (100-200 $\mu \mathrm{M}$ instead of $1 \mathrm{mM}$ ), buffer concentration (10 $\mathrm{mM}$ instead of $20 \mathrm{mM}$ ) and temperature $\left(25^{\circ} \mathrm{C}\right.$ instead of $\left.43^{\circ} \mathrm{C}\right)$. The slight differences in the wt c-kit2 NMR spectrum of the previously observed, ${ }^{40}$ and that in the current study, may be a consequence of different sample conditions.

We have therefore chosen to focus on the structural characterization of the dominant population and have obtained the assignments of the resolved imino proton resonances in G21T through site-specific ${ }^{15} \mathrm{~N}$-labelings (Figure 1a). ${ }^{44}$ With an apparent concentration of the ${ }^{15} \mathrm{~N}$-labeled guanines in each NMR sample of just $8 \mu \mathrm{M}$, the use of $2 \mathrm{D}^{15} \mathrm{~N}$ bandselective optimized flip-angle short transient heteronuclear multiple quantum coherence (SOFAST-HMQC) spectroscopy ${ }^{45}$ allows high resolution NMR data to be recorded within a few hours and effectively resolves all the ambiguities along the ${ }^{1} \mathrm{H}$ dimension due to partial overlaps for G8, G15 and G20 by separating the overlapped cross-peaks along the ${ }^{15} \mathrm{~N}$ dimension (Figure 1a). Notably, the imino groups of G2, G4 and G7 exhibit clear satellite peaks that have distinct chemical shifts along the ${ }^{1} \mathrm{H}$ dimension, and for $\mathrm{G} 2$ in particular, the ${ }^{15} \mathrm{~N}$ dimension. Together, these suggest the presence of a mixture of conformations in the NMR samples of G21T.

On the basis of the imino proton resonance assignments, we have assigned individual NOE correlations observed in the imino region of the 2D ${ }^{1} \mathrm{H}$ NOESY spectrum of G21T (Figure 1b). In addition, we have identified inter-strand NOEs (Figure 1d) that are consistent with a parallel G-quadruplex topology in which the four G strands are connected via two singlenucleotide propeller loops, loops 1 and 3 (L1 and L3), and a long loop 2 (L2) encompassing five nucleotides, CGCGA, through double chain-reversal (Figure 1c). Additionally, we have identified key NOE connectivities corresponding to interstrand correlations between imino proton $\mathrm{H} 1$ and aromatic proton $\mathrm{H} 8$ (Figure 1d). Of particular importance are the cross-peak of $\mathrm{G} 3(\mathrm{H} 1) \times \mathrm{G} 19(\mathrm{H} 8)$ between strands 1 and 4 (S1 and S4), and those of $\mathrm{G} 7(\mathrm{H} 1) \times \mathrm{G} 15(\mathrm{H} 8)$ 
and G8(H1)×G16(H8) between strands 2 and 3 (S2 and S3; boxed in Figure 1d), which essentially define the all-parallel topology together with the expected double chain-reversal configurations for $\mathrm{L} 1$ and $\mathrm{L} 3$. A much longer mixing time $(400 \mathrm{~ms})$ is required to obtain these key NOEs, in part due to the intrinsic dynamics of L2, and also the lack of covalent linkage to connect S1 and S4. Even with such a prolonged mixing time, the expected correlations of G14H8/G6H1 and G2H8/G18H1 from the top G-tetrad and that of G4H8/ G20H1 from the bottom G-tetrad remain unidentified. Additionally, after assigning most of the resolved (more than 260) through-space ${ }^{1} \mathrm{H}-{ }^{1} \mathrm{H}$ NOEs in the 2D NOESY spectra with various mixing times at different temperatures, we have been unable to identify a significant number of NOEs originated from L2, most likely due to conformational heterogeneity and/ or motions that lead to NMR line broadening. The cross-peaks G6(H1) $\times \mathrm{G} 2(\mathrm{H} 8), \mathrm{G} 7(\mathrm{H} 1)$ $\times \mathrm{G} 2(\mathrm{H} 8)$ and $\mathrm{G} 19(\mathrm{H} 1) \times \mathrm{G} 14(\mathrm{H} 8)$ in the NOESY spectra (Figure 1d) do not correspond to our unimolecular all-parallel-quadruplex and can be attributed to an alternative conformation that is also present in the solution. In order to clarify NOE assignments due to the contributions of mixed populations, we introduced nucleotide replacements in other loops, in addition to the G21T replacement.

\section{There Is Dynamical Coupling between the G-Tetrads and the Long Loop 2}

Four additional $c$-kit 2 variants were synthesized (Table 1), each of which contains a single nucleotide replacement in addition to the G21T replacement, for NMR spectral comparisons. Comparison of the $1 \mathrm{D}{ }^{1} \mathrm{H}$ spectra as well as the $2 \mathrm{D}{ }^{1} \mathrm{H}$ NOESY and TOCSY spectra of all the $c$-kit2 variants shows marginal spectral changes in terms of the relative chemical shift changes of the nonlabile protons, particularly for $\mathrm{H} 6 / \mathrm{H} 8$ in the bases and $\mathrm{H}^{\prime}{ }^{\prime}, \mathrm{H} 2^{\prime}$ and $\mathrm{H} 2^{\prime \prime}$ in the ribose moieties. The cytosine to thymine replacements in G21T/C9T and G21T/C11T enable immediate identification of the corresponding correlations between $\mathrm{H} 5$ and $\mathrm{H} 6$ in the spectra of G21T. Similarly, the assignments of the H2 and H8 protons of A17 have been confirmed by the disappearance of the most downfield shifted ${ }^{1} \mathrm{H}$ resonance at $8.5 \mathrm{ppm}$, corresponding to the $\mathrm{H} 8$ proton of the adenine base in G21T/A17T (data not shown). Interestingly, the imino proton spectra of individual c-kit2 variants exhibit marked differences both in chemical shift dispersions and in line widths, that is, dynamics (Figure 2). At $298 \mathrm{~K}$, all loop variants exhibit resolved imino proton resonances, most of which could be assigned by comparing the $2 \mathrm{D}^{1} \mathrm{H}$ NOESY spectra with respect to that of G21T. Note that these spectra were recorded after the overnight annealing. The cross-peaks G6(H1) $\times \mathrm{G} 2(\mathrm{H} 8), \mathrm{G} 7(\mathrm{H} 1) \times \mathrm{G} 2(\mathrm{H} 8)$ and $\mathrm{G} 19(\mathrm{H} 1) \times \mathrm{G} 14(\mathrm{H} 8)$, observed for G21T (Figure 1d), were absent in some of the loop variants (e.g. G21T/A17T, see below), suggesting that the second conformation was not significantly populated in these loop variants. In addition, $1 \mathrm{D}^{31} \mathrm{P}$ spectra of the loop variants show a similar peak distribution with a cluster centered at -1 $\mathrm{ppm}$, while the relative intensities and the degree of chemical shift dispersions are broadly correlated with the observations seen in the $1 \mathrm{D}^{1} \mathrm{H}$ spectra, suggesting some degree of structural differences at the backbone level also (data not shown). The differences in the folding kinetics between different loop variants may cause the differences in the overall appearance of the corresponding spectra at the particular time points when the spectra were recorded.

By changing the sample temperature from 298 to $283 \mathrm{~K}$ and $310 \mathrm{~K}$, followed by a brief equilibration period of ca. 10 min inside the spectrometer, the loop variants exhibit significant line-broadening for most $c$-kit2 variants, except for G21T/A17T, suggesting conformational changes taking place on the millisecond (ms) timescale. Given that the very slow refolding kinetics of wt and G21T is on a timescale between days and months (Figure $\mathrm{S} 1$ ), the observed broadening could be a result of local rearrangements that have been slowed down at a lower temperature. At the elevated temperature of $310 \mathrm{~K}$, wt c-kit2 exhibits 12 well-resolved resonances (bearing in mind that this particular sample has been 
equilibrated extensively; Figure S1) with roughly equal peak intensities. A comparable improvement is observed for G21T/G10A. The spectral qualities of G21T/C9T and G21T/ C11T, however, deteriorate somewhat compared to those obtained at $298 \mathrm{~K}$. It was noteworthy that the 1D imino proton spectra of the loop variants, in particular G21T/A17T, recorded at 298K resemble the initial spectrum (recorded within $24 \mathrm{~h}$ ) of G21T (Figures 2 and S1) more than that of an aged G21T (recorded after several weeks; Figure S1) bassed on which we assigned the individual imino proton resonances through ${ }^{15} \mathrm{~N}$ enrichments (Figure 1a).

Among all the resolved imino proton resonances, G2 H1 has the most downfield chemical shifts for all c-kit 2 variants with much higher sensitivity toward temperature changes for G21T/G10A and slightly less so for G21T/C9T and G21T/C11T (Figure 2). The G2 H1 resonances of all loop variants but G21T/G10A also show clear doubling as observed in the initial spectra of wt and G21T (Figures 1 and 2). For G21T/G10A, the resonances of G8 H1 and G19 H1 show pronounced upfield shifts by ca. $0.5 \mathrm{ppm}$ in comparison to those of G21T. The chemical shifts and line-shapes of these resonances are not sensitive to temperature changes, suggesting a well-defined conformation, involving S2 and S4, which is rather unexpected because the two imino protons are not involved in the same G-tetrad. Given the well-resolved imino proton spectrum of G21T/G10A, we decided to carry out further comparative spectral analyses for G21T and G21T/G10A. The common features consistently observed in all loop variants were used in our subsequent structural elucidation of the unimolecular all-parallel topology.

NMR-based structural characterization relies heavily on the resonance assignments of NOE correlations and the subsequent conversion of cross-peak intensities to distance restraints. ${ }^{46}$ As mentioned above, the NOESY spectrum of G21T exhibits a large dynamic range, differences in the spin-relaxation properties due to dispersed internal motions, reflected in line widths, with many expected loop resonances missing, an observation that we have attributed to conformational variability and differences in dynamics between different parts of the molecule. For example, the intrabase $\mathrm{H} 5 / \mathrm{H} 6$ in cytosines has a fixed distance of 2.6 $\AA$, which is expected to give rise to an intense NOEs. The intensities of the H5/H6 crosspeaks of $\mathrm{C} 9$ and $\mathrm{C} 11$, however, are about 1/10 of that of C5 and C11 in G21T and are much weaker than many of the intraresidue $\mathrm{H} 8 / \mathrm{H} 1^{\prime}$ correlations (Figure $3 \mathrm{a}$ ). The differences in the observed peak heights of the same $\mathrm{H} 5 / \mathrm{H} 6$ cross-peaks, that is, dynamic range, are reduced in G21T/G10A, while the chemical shifts of most resonances exhibit marginal changes.

Additionally, a number of residues, including C9, A10 and G19, exhibit clear peak doubling with similar peak intensities, suggesting the presence of another similar but distinct conformation. Finally, the intraresidue correlations of G14 H8/H1 and A13 H8/H2 could now be identified through additional correlations with C11 in other regions of the NOESY spectrum (Figure 3b). Importantly, the relatively weak intraresidue $\mathrm{H} 8 / \mathrm{H}^{\prime}$ ' peak intensities have led us to conclude that all the tetrad-forming guanines are in anti conformations ${ }^{47}$ (G8 and G18 are somewhat ambiguous as the corresponding peak intensities are not as weak as other ones, which could again be attributed to the intrinsic dynamics; see below). Together with the H1-H1 (Figure 1) and H1-H1' (not shown) correlations, we have established the folding topology of $c$-kit 2 variants to be in a parallel topology with double chain reversals for all connecting loops and the nucleotides in all three G-tetrads in anti anti anti - anti conformations. ${ }^{2}, 3$

\section{Solution Structures of the c-kit2 Variants All Adopt a Parallel Propeller Topology}

We have generated models for the average structure of $c$-kit 2 variants, G21T and G21T/ G10A, using the experimentally derived distance restraints. The molecules have all been assigned a parallel topology with three G-tetrads (Figure 4) and three chain-reversal (propeller) loops. L1 and L3 are single-nucleotide (C5 and A17, respectively), and L2 is a 
pentanucleotide loop (CGCGA in wt c-kit2). Considerable variability in the structure of the loops was seen, particularly the pentanucleotide loop, in part as a consequence of the lack of experimental distance restraints. The terminal bases, $5^{\prime} \mathrm{C} 1$ and $3^{\prime} \mathrm{T} 21$, stack on either side of the G-quadruplex core to cap the terminal tetrad planes: C1 against G2/G6 and T21 against G20. Although both L1 and L3 are single-nucleotide loops, the structure of C5 is much less defined, and the cytosine base adopts a wide range of orientations, consistent with the very intense H5/H6 cross-peak that indicates significant internal mobility (Figure 3). A17, on the other hand, has a much more defined conformation and it is pointing toward the backbone of S4 (Figure 4a,c). The long L2 is very flexible as also evidenced by the presence of very few associated NOEs. However, there is a propensity to form an internal stacking arrangements within the loop. In G21T/G10A, the mutated A10 is often found to be stacked against $\mathrm{C} 9$ and $\mathrm{C} 11$ to form a more complex loop structure. Direct stacking between $\mathrm{C} 9$ and $\mathrm{C} 11$ has also been observed. The other two loop residues, G12 and A13, are very flexible, but G12 is able to stack on the top G-tetrad and hydrogen bond to C1. Altogether, the loop behaves as a short and highly flexible single strand of DNA with a tether at each end.

\section{NMR Hydrogen-Deuterium Exchange Experiment Reveals Hydrogen Bonding Asymmetry in the G-Tetrads}

To investigate the dynamical differences between the loop variants of $c$-kit2, we have carried out NMR hydrogen-deuterium exchange (HDX) experiments to extract the kinetics of solvent-mediated exchanges of the imino protons of $c$-kit 2 variants under equilibrium conditions. The rates of HDX $\left(k_{\mathrm{HDX}}\right)$ reflect the degree of local fluctuations, that is, dynamics, of individual hydrogen bonds within the G-tetrads. Such information has been widely used to extract the free energy of unfolding at a residue-specific level for proteins. 48,49 For nucleic acids, the lifetimes of hydrogen bonded imino protons in Watson-Crick base-paired duplexes are on the timescale of $1-50 \mathrm{~ms},{ }^{50}{ }_{-5} 52$ imino protons in higher order structures such as tRNA ${ }^{53}$ and triplexes ${ }^{54}$ exchange much more slowly, which are on the timescale of hours to days. For G-quadruplexes, the degree of protection for imino protons within the G-tetrads is much higher and the corresponding exchange rates are much slower. Phan et al. have reported ${ }^{19}$ the rates of HDX for the four most protected imino protons of $m y c-2345$ and $m y c-1245$ to be in the range of $15-40 \mathrm{~h}$, that is, an exchange rate $k_{\mathrm{HDX}}$ of ca. $1 \times 10^{-5} \mathrm{~s}^{-1}$. Few studies on G-quadruplexes so far, however, have reported on the imino proton exchange kinetics; HDX can be employed to identify the most protected imino protons, usually the four in the central G-tetrads with similar degree of protection, by recording the corresponding imino proton spectra at a single time point after incubating the samples in $\mathrm{D}_{2} \mathrm{O}$ for typically $4-24$ h. ${ }^{6,},{ }^{4}, 55-59$

Given the abundant internal dynamics of $c$-kit 2 observed in this work, we expected to see a broader distribution of HDX profiles of the 12 imino protons of individual $c$-kit 2 variants. Immediately after dissolving the lyophilized c-kit2 variants into $\mathrm{D}_{2} \mathrm{O}$, between six and 10 imino proton resonances were identified in the first $1 \mathrm{D}^{1} \mathrm{H}$ NMR spectrum with G2 H1 being the least protected, which is consistent with its higher temperature sensitivity as described above. Additionally, G3 H1 and G7 H1 are the most protected of all, with a significant level of residual signals over $20 \mathrm{~h}$ of HDX for all loop variants; those of G21T/ A17T exchange at slightly faster rates, resulting in a complete exchange within $20 \mathrm{~h}$. The use of a $900 \mathrm{MHz}$ NMR spectrometer allows highly resolved imino proton resonances to be obtained, which is critical for signal integration as a function of HDX time for subsequent data fitting. This has been particularly successful for G21T/G10A with all resonances exhibiting baseline separation (Figure 5). Additionally, the peak doublings of G3 H1 and G7 $\mathrm{H} 1$ remain visible in the spectrum of G21T/C9T after $20 \mathrm{~h}$ of HDX (Figure 5a). Note that the relative peak heights between the doublets are different at time 0 and after $20 \mathrm{~h}$. We have 
assigned the identities of the fast exchanging ones as $\mathrm{G}^{\prime}{ }^{\prime}$ and $\mathrm{G}^{\prime}$, and the slow exchanging ones as G3 and G7 hereafter (Figure 5a).

HDX under equilibrium conditions is expected to give rise to single-exponential decaying profiles for individual exchangeable groups. In the case of $c$-kit2, the HDX profiles for the slow-exchanging imino protons, particularly those of G3, G7, G15 and G19 in the central Gtetrad, are clearly double-exponential with a fast HDX rate, and a slower one that differs by at least 10-fold; the initial amplitudes of the two were comparable in magnitude (Table 2 and Experimental Section). The results suggest the presence of two distinct dynamic states that have very similar structures in NMR terms, that is, similar chemical shifts. In the case of G21/C9T, two distinct resonances have been identified and assigned to the imino protons of G3 and G3' (minor conformation of G3). As the two resonances were significantly overlapped, they were thus integrated together and subject to rate analysis, giving rise to two exchange rates, $35 \times 10^{-6}$ and $1035 \times 10^{-6} \mathrm{~s}^{-1}$, with similar initial amplitudes (Table 2).

Assuming that the two populations independently exchange with bulk solvent, the resonance corresponding to the fast exchanging population will decay beyond detection after $20 \mathrm{~h}$, which is in contrast to the experimental observation (Figure 5a). It is therefore plausible that an additional interconversion process exists between the slow and fast exchanging populations that leads to a significant fraction of the initial population, which is in the slow exchanging population, to switch to the fast exchanging conformation and vice versa. Hence, the apparent intensities of the two populations are comparable, as a result of the interconversion during the course of the HDX process.

Comparison of the observed $k_{\mathrm{HDX}}$ values of all c-kit2 variants shows that, in general, G3 H1 and $\mathrm{G} 7 \mathrm{H} 1$ in the first two strands are the most protected imino protons and that, for G21T/ C9T and G21T/G10A, G15 H1 and G19 H1 are exchanging ca. 3-5 times faster than the

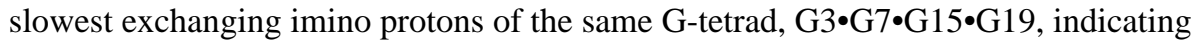
dynamical asymmetry within the core structure, which is in contrast with most of the previous NMR findings that the four imino protons in the central G-tetrads are equally protected during HDX. ${ }^{6},{ }^{47}, 55-59$ The slowest exchanging imino group, G7 $\mathrm{H} 1$ for most $c$ kit 2 variants, have a half time, $t_{1 / 2}$, of ca. $10 \mathrm{~h}$, which is at the lower bound of what has been reported for the $c$-myc G-quadruplex, which is in the range of $10-50 \mathrm{~h},{ }^{19}$ suggesting that the central G-tetrad of $c$-kit2 fluctuates more than that of $c$-myc. Additionally, G4 H1 and G6 $\mathrm{H} 1$ that flank the single nucleotide $\mathrm{L} 1$ also exhibit a higher degree of protection in comparison with those that flank L3, namely, G16 H1 and G18 H1.

\section{Discussion}

There is good evidence from a large number of studies to suggest that a given G-rich nucleic acid sequence can adopt a variety of G-quadruplex folding topologies often accompanied by the ability to interconvert between these states over a broad range of time scales. Depending on the conditions used for structural investigations, one of several conformations can be preferentially populated. ${ }^{60},{ }^{61} \mathrm{We}$ and others have used single molecule fluorescence spectroscopy to demonstrate that human telomeric DNA G-rich repeats can adopt multiple G-quadruplex conformations, both parallel and antiparallel, in dilute solution with temperature- and counterion-dependent folding and unfolding rates; ${ }^{62}{ }^{64}$ similar results have been derived by solution NMR spectroscopy. ${ }^{57},{ }^{65},{ }^{66}$ In the case of $c$-kit2, we have also shown that the wt sequence exhibits two distinct sets of FRET, NMR and CD signals in the absence (or presence of low concentrations) of potassium ions, which we tentatively attributed to the coexistence of parallel and antiparallel conformations. ${ }^{43},{ }^{67} \mathrm{We}$ have confirmed in the present study that $c$-kit 2 forms a parallel quadruplex in $\mathrm{K}^{+}$solution with internal dynamics that are modulated by loop composition. ${ }^{16}, 68,69$ 
Among a handful of intramolecular G-quadruplex structures that have been reported so far, the majority has been those of human telomeric DNA repeats with relatively long loops and hence much more polymorphic. ${ }^{4}{ }^{6}$ Meanwhile, intramolecular promoter G-quadruplex often have shorter loops. ${ }^{14},{ }^{17},{ }^{39},{ }^{40},{ }^{70}$ On the basis of the sequence of $c$-kit2 G21T, one could expect parallel arrangements for the first two (S1 and S2) and last two strands (S3 and S4), both of which are connected by a single nucleotide $\left(\mathrm{G}_{3} \mathrm{NG}_{3}\right)$, which strongly favors double chain reversal. ${ }^{16}, 68,69$ G-quadruplexes in human $c$-myc promoter, myc-2345 ${ }^{19}$ and MYC22$\mathrm{G} 14 \mathrm{~T} / \mathrm{G} 23 \mathrm{~T},{ }^{20}$ have been found to adopt an all-parallel topology with short loops ( $5^{\prime}$ $\mathrm{G}_{3} \mathrm{NG}_{3} \mathrm{~N}_{2} \mathrm{G}_{3} \mathrm{NG}_{3}-3^{\prime}$ ). Although L3 in wt $c$-kit2 could in principle be two-nucleotides long when G19-G21 are involved in the G-tetrad formation, a single nucleotide L3 (A17 alone) is kinetically more favorable as has been demonstrated by a systematic survey of model oligonucleotides. ${ }^{71}$ The relatively long and unstructured L2 loop provides the additional degree of freedom necessary for $c$-kit 2 in principle to adopt either an all-parallel, propellertype conformation, or a mixed parallel/antiparallel conformation with a long lateral loop (Figure 6a). In the case of $m y c-1245,{ }^{19}$ which has a $\mathrm{T}_{4}$ replacement for the third G-tract of the five consecutive G-tracts in the wt $c$-myc sequence, it was found to be in an all-parallel topology with a hexanucleotide loop connecting $\mathrm{S} 2$ and $\mathrm{S} 3\left(5^{\prime}-\mathrm{G}_{3} \mathrm{NG}_{3} \mathrm{~N}_{6} \mathrm{G}_{3} \mathrm{NG}_{3}-3^{\prime}\right)$. In contrast, the G-quadruplex formed in human $b c l-2$ promoter $^{70}$ was found to be in a mixed parallel/antiparellel topology with a largely disordered heptanucleotide loop connecting S2 and $\mathrm{S} 3\left(5^{\prime}-\mathrm{G}_{3} \mathrm{NG}_{3} \mathrm{~N}_{7} \mathrm{G}_{3} \mathrm{NG}_{3}-3^{\prime}\right)$.

The diversity of topologies that can be adopted by G-quadruplexes can be best appreciated by comparing the structure of $c$-kit 2 and that of $c$-kit 1 , which are found proximally within the same promoter. Both have all strands parallel, but the former has three conventional strand-reversal loops, whereas the latter has two strand-reversal loops together with a dinucleotide loop connecting two adjacent corners and a pentanucleotide loop that folds back to allow a terminal guanine to participate in the tetrad core. ${ }^{36}$ The pentanucleotide stem loop of c-kit1 (of sequence AGGAG) is highly structured compared to the loop in c-kit2, since it is stabilized by stacked A - G base pairs. The relatively unconstrained all-parallel topology may be the origin of the dynamical asymmetry that has been observed for all variants of $c$-kit 2 delineated from the HDX data.

Having established the assignments of the imino proton resonances of all the loop variants of $c$-kit2, we have been able to assign those of the wt with 12 well-resolved resonances, denoted as the low energy state (lower panel in Figure 6b). Comparing the similarities of these resonances with respect to the initial spectrum of wt, a number of peaks in the initial spectrum could be tentatively assigned. G2, G3 and G7 have very similar chemical shifts in the initial spectrum to those in the low energy state. Additionally, G6 and/or G14 also exhibit the low energy state-like chemical shifts in the initial spectrum. Collectively, these residues are located in one corner of the three-layer G-tetrad core structure (Figure 6c). This could be rationalized by S4, particularly G20 and G21, tending to switch their G-tetrad registering in a latch sliding manner, ${ }^{72}$ leading to fluctuations in the bottom G-tetrad as well as in the local arrangements of S3-L3-S4. In the low energy state, G8, G15, G16, G18, and G20 in the other half of the core structure exhibit well-resolved upfield shifted lines $\left(\delta^{1} \mathrm{H}\right.$ $10.7-11.2 \mathrm{ppm}$ ), while a collapsed broad line is observed in the initial spectrum as a result of conformational heterogeneity. Although it is assumed that a single nucleotide double chain reversal is kinetically the most favorable conformation for S3-L3-S4 within each topology, $16,68,69 \mathrm{~L} 3$ can also be a double nucleotide one in the wt sequence (consists of A17 and G18). The exceedingly slow folding kinetics observed for wt $c$-kit2 may be the result of multiple parallel folding pathways in a rugged energy surface, rather than sequential folding events of obligatory intermediate formation with very high kinetic barriers. 
Our NMR HDX experiments have provided intriguing evidence of the existence of dynamical asymmetry within the G-tetrads: the first half of the molecule, S1-L1-S2, is slightly better defined (less local fluctuations) than is the second half, S3-L3-S4. It is also apparent that there are at least two populations present in solution that are structurally very similar, yet distinguishable by NMR spectroscopy. The fact that two sets of NMR resonances, separated by very small chemical shift differences, have been identified for a number of G-tetrad forming residues (Figures 1,2 and 5) suggests that the two conformations are in the same parallel conformation and that the exchange between the two states, if indeed present, is slow on the NMR timescale $\left(>\mathrm{s}^{-1}\right)$. Indeed, previous NMR studies on myc-2345 and myc-1245 have shown that the imino proton chemical shifts (local structures) are not sensitive to a long loop extension in myc-1245, but the rates of HDX for the imino protons (dynamics) within the central G-tetrad of myc-1245 are ca. 2 times faster compared to those of myc-1245. There was, however, no evidence of internal dynamics asymmetry nor conformational interconversion in this study. Here, the HDX data on G21T/ C9T (Figure 5a) shows the possible interconversion between the two states as the doublet imino proton signals of $\mathrm{G} 3$ and $\mathrm{G}^{\prime}$, as well as those of $\mathrm{G} 7$ and $\mathrm{G}^{\prime}$, decay at a similar rate indicating that the pool of the fast exchanging imino protons is replenished by the slow exchanging ones during the apparently irreversible HDX process (Figure $5 b$ ). This is reminiscent of the interconversions between short-lived and long-lived human telomeric DNA G-quadruplexes observed by single molecule FRET measurement ${ }^{64}$ with corresponding lifetimes in the order of 10 and $100 \mathrm{~s}$, respectively. The relatively fast exchange process at equilibrium suggests that the conformations are similar in energy and are separated by relatively small kinetic energy barriers to allow redistribution of populations during the course of HDX on the timescale of $10^{3}-10^{6} \mathrm{~s}$.

Genomic DNA sequences exist predominantly in a duplex form, but must necessarily be unwound into a single-stranded form to enable transcription. Thus, in vivo, duplex versus quadruplex stability may be further influenced by the local environment that will include factors such as superhelicity. ${ }^{23},{ }^{73}$ G-quadruplex forming sequences in the $5^{\prime}$ promoter regions of human genome and formation of higher order structures may play an important role in transcription regulation, particularly those that are associated with oncogenesis. $7,9,74$ Akin to protein folding, G-quadruplex formation is a highly cooperative process. ${ }^{60}$ The folding pathways of G-quadruplexes, however, can consist of a large number of pathways with the potential to switch from one to another depending on the sample conditions. We have shown here that, where the presence of potassium ions and the G21T replacement favor a parallel conformation, $c$-kit 2 exits in at least two subtly distinct conformational states that are structurally and energetically similar. These are most likely substates populated in the global minimum, which would take a very long period of time for wt $c$-kit 2 to reach due to the complexity caused by the additional guanine at the $3^{\prime}$ end (Figure 1). The HDX data on its own, however, cannot provide information about the folding pathways of G-quadruplex formation without the help of further kinetics information. ${ }^{49},{ }^{75}$ What it has demonstrated, however, is how sensitive the dynamics and stabilities are in response to the subtle structural differences manifested in small NMR chemical shift changes, for example, G21T/G10A (Figure 5a), and most likely indistinguishable by UV, CD and FRET measurements.

It remains to be seen as to how these low energy substates can be attained from a fully extended structure with the competition of a complementary strand. It has also been shown that a number of small molecules can target specific loop structures ${ }^{76}$ with the potential to stabilize quadruplex structures. The L2 loop of $c$-kit 2 would be an ideal structural feature to target for recognition with the aim of selecting specific conformations through ligand binding. ${ }^{77}$ 


\section{Experimental Section}

\section{Labeled Oligodeoxyribonucleotide Preparation}

The labeled oligodeoxyribonucleotide sequences were synthesized on an Applied Biosystems DNA synthesizer using solid-phase $\beta$-cyanoethylphosphoramidite chemistry.

Bz-dA-CE phosphoramidite, iBu-dG-CE phosphoramidite, and dT-CE phosphoramidite were supplied by Sigma-Aldrich Co. Ltd., U.K., and Ac-dC-CE phosphoramidite by Link Technologies Ltd., U.K. (U- ${ }^{13} \mathrm{C}_{10}, 98 \%$; U- ${ }^{15} \mathrm{~N}_{5}, 98 \%$ ) iBu-dG-CE phosphoramidite, manufactured by Cambridge Isotope Laboratories, Inc., was obtained from CK GAS Products Ltd., U.K. Dilution of the labeled iBu-dG-CE phosphoramidite ( $15 \mathrm{mg}, 17.5 \mu \mathrm{mol})$ was carried out by injecting a solution of the unlabeled $\mathrm{G}$ phosphoramidite in acetonitrile $(0.0916 \mathrm{M}, 2.1 \mathrm{~mL})$ into the supplied vial, resulting in a $1.0 \mathrm{M}$ solution of $8 \%\left[{ }^{13} \mathrm{C},{ }^{15} \mathrm{~N}\right]$ enriched phosphoramidite. To make more efficient use of the labeled phosphoramidite, a polymeric T resin (dT OligoPrep 250 PVA support - Link Technologies Ltd., U.K., specific loading $253 \mu \mathrm{mol} / \mathrm{g}$ ) was used rather than standard CPG support. Reusable synthesis columns were loaded with $12 \mathrm{mg} \mathrm{(} 3 \mu \mathrm{mol})$ of support and preswelled with acetonitrile for 1 $\mathrm{h}$ immediately before use. The standard $1 \mu \mathrm{mol}$ synthesis cycle was used for synthesis after being edited by prolonging phosphoramidite coupling times, and by increasing the volumes and reaction times of the deblocking, capping, and oxidizing reagents.

The oligodeoxyribonucleotides were cleaved from the support and deprotected by passing a 1:1 mixture of 0.88 ammonia and $40 \%$ aqueous methylamine back and forth through the synthesis columns for $10 \mathrm{~min}$ and then heating the solutions at $60{ }^{\circ} \mathrm{C}$ for $30 \mathrm{~min}$ in sealed microcentrifuge tubes. The ammonia and methylamine were removed using a centrifugal evaporator and the crude oligodeoxyribonucleotides dissolved in ammonium acetate buffer (100 mM, pH 10.0). Purifications were carried out by HPLC on a BioCAD Workstation with an Oligo R3 column (PerSeptive Biosystems, Inc.). The samples were then dialyzed against double-distilled water, yields determined by measuring the absorbance of the dialyzed oligodeoxyribonucleotide solutions at $260 \mathrm{~nm}$, and lyophilized with a centrifugal evaporator to produce the final purified products.

\section{NMR Spectroscopy for Resonance Assignments}

All NMR samples have been subject to an annealing process by heating the NMR sample solutions at $95{ }^{\circ} \mathrm{C}$ for $5 \mathrm{~min}$ and slowly cooled to room temperature. The unlabeled wt and loop variants were synthesized and purified by double HPLC through a commercial source (IBA GmBH, Germany). All NMR samples, including the site-specifically ${ }^{13} \mathrm{C} /{ }^{15} \mathrm{~N}$ labeled samples, contain oligo concentrations of ca. $100 \mu \mathrm{M}$, buffered in $10 \mathrm{mM}$ potassium phosphate ( $\mathrm{pH} 7.0)$, and 7\% $\mathrm{D}_{2} \mathrm{O}(\mathrm{v} / \mathrm{v})$ as an NMR lock signal. All NMR data have been recorded at the ${ }^{1} \mathrm{H}$ Lamor frequencies of 500, 700, and $900 \mathrm{MHz}$ with NMR spectrometers equipped with cryogenic probes (Bruker, Germany). 1D imino proton spectra of all samples were recorded using the jump-and-return (JR) scheme to minimize saturation of the labile NMR signals as a result of water suppression. ${ }^{78} 2 \mathrm{D}{ }^{1} \mathrm{H}-{ }^{15} \mathrm{~N}$ SOFAST-HMQC spectra ${ }^{45}$ of 12 site-specifically ${ }^{15} \mathrm{~N}$-labeled NMR samples of the G21T variant were recorded to obtain unambiguous resonance assignments: each NMR sample contains $8 \%\left[{ }^{13} \mathrm{C},{ }^{15} \mathrm{~N}\right]$ labeled guanine base introduced to one of the $12 \mathrm{G}$-tetrad forming guanine residues as described above; each NMR sample is designated as $\left[8 \%-{ }^{13} \mathrm{C} /{ }^{15} \mathrm{~N}, \mathrm{G}_{\mathrm{i}}\right]-\mathrm{G} 21 \mathrm{~T}$ to indicate the labeling site $i\{i=2,3,4,6,7,8,14,15,16,18,19,20\}$. Additionally, ${ }^{13} \mathrm{C}$-edited $1 \mathrm{D}{ }^{1} \mathrm{H}$ spectra were recorded for all $12\left[8 \%-{ }^{13} \mathrm{C} /{ }^{15} \mathrm{~N}, \mathrm{G}_{\mathrm{i}}\right]-\mathrm{G} 21 \mathrm{~T}$ samples to obtain $\mathrm{H} 8$ resonance assignments of individual guanines within the G-tetrad. Resonance assignments of the remaining protons have been determined through a combination of $2 \mathrm{D}^{1} \mathrm{H}$ NOESY with 
various mixing times ranging from 50 to $400 \mathrm{~ms}, 2 \mathrm{D}{ }^{1} \mathrm{H}$ TOCSY with a mixing time of 60 $\mathrm{ms}$, and $2 \mathrm{D}{ }^{1} \mathrm{H}$ DQF-COSY spectra. ${ }^{78}$

\section{Molecular Modeling}

Two sequences, corresponding to G21T (dCGGGCGGGCGCGAGGGAGGGT) and G21T/ G10A (dCGGGCGGGCACGAGGGAGGGT) mutants of $c$-kit2 were generated in ideal extended conformation using the leap module of Amber $10 .{ }^{79}$ In addition, the same sequences were built into a parallel quadruplex topology using the crystal structure of the human telomeric quadruplex (PDB accession ID: $1 \mathrm{KF} 1)^{5}$ as a template. Both starting models were subjected to a restrained simulated annealing conformational search protocol to evaluate the convergence of the final ensembles using the simulation package Amber10. ${ }^{79}$

\section{NOE Restraints}

All assigned NOESY cross-peaks were classified to strong (1.8-2.8 $\AA$ ), medium (1.8-3.6 $\AA$ ), weak (1.8-5.5 $\AA$ ) and very weak (1.8-6.5 $\AA$ ) interproton distance restraints according to their intensities. Cross-peaks related to the thymine methyl group were interpreted as ambiguous restraints and the $\left\langle r^{-6}\right\rangle-1 / 6$ averaged values of the three equivalent distances was restrained. Beyond these bounds, a quadratic penalty potential was applied with a force constant of $10 \mathrm{kcal} \mathrm{mol}^{-1} \AA^{-2}$. A total of 236 distance restraints were used for G21T and 300 distance restraints were used for G21T/G10A. In addition, ideal hydrogen bond restraints were imposed on the guanine bases in the anti anti antio anti tetrad core using two distances per hydrogen bond. Donor to acceptor distance was set to $2.8-3.0 \AA$ and proton to acceptor distance to 1.8-2.0 $\mathrm{\AA}$ with a force constant of $50 \mathrm{kcal} \mathrm{mol}^{-1} \AA^{-2}$. Bases corresponding to a G-tetrad were restrained to lie in the same plane with a force constant of $50 \mathrm{kcal} \mathrm{mol}^{-1} \mathrm{rad}^{-2}$. The glycosylic $\chi$ angle $\left(\mathrm{O}^{\prime}-\mathrm{Cl}^{\prime}-\mathrm{N} 9-\mathrm{C} 4\right)$ was restrained to an anti conformation $\left(240 \pm 70^{\circ}\right)$ for all guanines participating in the G-quadruplex tetrad with a force constant of $50 \mathrm{kcal} \mathrm{mol}^{-1} \AA^{-2}$.

For restrained simulated annealing calculation, initial models were energy minimized without restraints and subjected to 200 simulated annealing cycles using the full set of restraints. A cycle started from the structure obtained in the previous cycle and included 10 ps heating from 300 to $1000 \mathrm{~K}$ followed by an equilibration of $10 \mathrm{ps}$ at $1000 \mathrm{~K}$ without any restraints applied. The force constants for all restraints were then scaled gradually from zero to the final values during $20 \mathrm{ps}$ at $1000 \mathrm{~K}$ followed by cooling the system to $300 \mathrm{~K}$ over 30 ps. Interactions within the system were calculated using the latest Amber force field for nucleic acids (ff99bsc 0$)^{80}, 81$ and Generalized Born solvation $(i g b=2)$ with monovalent salt concentration corresponding to $0.1 \mathrm{M}$. The final structures were energy minimized using the same parameters and restraints described above. Ten best models for an average structure were selected based on the value of the overall potential energy and NMR restraint violations to undergo further refinement in explicit water and counterion environment.

For structure refinement with molecular dynamics in explicit solvent, each final model structure was placed in a truncated octahedral box of about 5000 water molecules and $20 \mathrm{~K}^{+}$ counterions. Two $\mathrm{K}^{+}$ions were inserted in the core of the quadruplex with ideal coordination geometry between stacked guanine tetrads as seen in the crystal structure (PDB accession ID: 1KF1). Potassium ions were maintained at this position throughout using distance restraints (2.7-3.0 $\AA$ ) to O6 of the corresponding guanines with force constant of 10 kcal mol ${ }^{-1} \AA^{-2}$. The system was energy minimized and then heated to $300 \mathrm{~K}$ at constant volume during $50 \mathrm{ps}$ while the quadruplex was kept under positional restraints with force constant of $25 \mathrm{kcal} \mathrm{mol}^{-1} \AA^{-2}$. The positional restraints were gradually removed over $300 \mathrm{ps}$ at constant pressure $(1 \mathrm{~atm})$ and temperature $(300 \mathrm{~K})$ and a production phase was initiated for $2 \mathrm{~ns}$ with the full set of restraints applied, but the planarity and hydrogen bond restraints 
in the G-tetrad reduced to $5 \mathrm{kcal} \mathrm{mol}^{-1} \AA^{-2}$. The final structures were energy minimized and used for subsequent analysis. The coordinates of the ensembles of G21T and G21T/G10A have been deposited in the Protein Data Bank with the accession IDs of 2KJ2 and 2KJ0, respectively.

\section{NMR Hydrogen-Deuterium Exchange (HDX) Experiments}

A total of $500 \mu \mathrm{L}$ of each NMR sample in aqueous solution was flash frozen in liquid nitrogen and subsequently lyophilized overnight prior to the HDX experiments. A total of $500 \mu \mathrm{L}$ of $99 \% \mathrm{D}_{2} \mathrm{O}$ was added to dissolve the lyophilized sample immediately before each NMR measurement using a $900 \mathrm{MHz}$ NMR spectrometer. A series of $1 \mathrm{D}^{1} \mathrm{H}$ NMR spectra were recorded until most imino proton signals have significantly attenuated after typically 14-20 h. HDX under NMR conditions follows a pseudo-first-order kinetics. The observed imino proton resonances were integrated and analyzed as a function of HDX time and fitted to the formula below,

$$
I(t)=A_{0}+A_{\mathrm{s}} e^{\left(-k_{\mathrm{HDX}}^{\mathrm{s}} \times t\right)}+A_{\mathrm{f}} e^{\left(-k_{\mathrm{HDx}}^{\mathrm{f}} \times t\right)}
$$

where $A_{0}$ is the baseline given by the residual $\mathrm{H}_{2} \mathrm{O}$ content, $A_{\mathrm{s}}$ and $A_{\mathrm{f}}$ are the amplitudes of two distinguishable decaying phases to the integrated NMR signals, respectively; $k_{\mathrm{HDX}}^{\mathrm{s}}$ and $k_{\mathrm{HDX}}^{\mathrm{f}}$ are the rate constants corresponding to the major and minor (slow and fast) contributions, respectively. Note that a single exponential decaying function with baseline is used when the two rates are similar, $k_{\mathrm{HDX}}^{\mathrm{s}} k_{\mathrm{HDX}}^{\mathrm{f}}$, or when one amplitude is insignificant to the overall amplitude, $A_{\mathrm{s}} \gg A_{\mathrm{f}}$ or $A_{\mathrm{s}} \ll A_{\mathrm{f}}$, to avoid overfitting. The data fittings were carried out using the built-in module within the program Grace (http://plasma-gate.weizmann.ac.il/ Grace/).

\section{Acknowledgments}

We thank the BBSRC for project funding, Cancer Research UK for programme funding (to S.B. and S.N.). S.T.D.H. is a recipient of a Human Frontier Long-term Fellowship (LT0798/2005) and is supported in part by the Postdoctoral Research Abroad Program of National Science Council of the Republic of China, Taiwan (NSC97-2917-1-564-102). We thank the staff and the use of the Biomolecular NMR Facility, Department of Chemistry, University of Cambridge. The $900 \mathrm{MHz}$ NMR spectra were recorded at the SON NMR Large Scale Facility in Utrecht, The Netherlands (funded by the European Union, contract number RII3-026145).

\section{References}

1. Davis JT. Angew. Chem., Int. Ed. Engl. 2004; 43:668-98. [PubMed: 14755695]

2. Burge S, Parkinson GN, Hazel P, Todd AK, Neidle S. Nucleic Acids Res. 2006; 34:5402-15. [PubMed: 17012276]

3. Patel DJ, Phan AT, Kuryavyi V. Nucleic Acids Res. 2007; 35:7429-55. [PubMed: 17913750]

4. Wang Y, Patel DJ. J. Mol. Biol. 1993; 234:1171-83. [PubMed: 8263919]

5. Parkinson GN, Lee MP, Neidle S. Nature. 2002; 417:876-80. [PubMed: 12050675]

6. Luu KN, Phan AT, Kuryavyi V, Lacroix L, Patel DJ. J. Am. Chem. Soc. 2006; 128:9963-70. [PubMed: 16866556]

7. Huppert JL, Balasubramanian S. Nucleic Acids Res. 2005; 33:2908-16. [PubMed: 15914667]

8. Todd AK, Johnston M, Neidle S. Nucleic Acids Res. 2005; 33:2901-7. [PubMed: 15914666]

9. Huppert JL, Balasubramanian S. Nucleic Acids Res. 2007; 35:406-13. [PubMed: 17169996]

10. Verma A, Halder K, Halder R, Yadav VK, Rawal P, Thakur RK, Mohd F, Sharma A, Chowdhury S. J. Med. Chem. 2008; 51:5641-9. [PubMed: 18767830]

11. Todd AK, Neidle S. Nucleic Acids Res. 2008; 36:2700-4. [PubMed: 18353860] 
12. Neidle S. Curr. Opin. Struct. Biol. 2009; 19:239-50. [PubMed: 19487118]

13. Qin Y, Hurley LH. Biochimie. 2008; 90:1149-71. [PubMed: 18355457]

14. Dai J, Chen D, Jones RA, Hurley LH, Yang D. Nucleic Acids Res. 2006; 34:5133-44. [PubMed: 16998187]

15. Hazel P, Parkinson GN, Neidle S. J. Am. Chem. Soc. 2006; 128:5480-7. [PubMed: 16620121]

16. Bugaut A, Balasubramanian S. Biochemistry. 2008; 47:689-97. [PubMed: 18092816]

17. Simonsson T, Pecinka P, Kubista M. Nucleic Acids Res. 1998; 26:1167-72. [PubMed: 9469822]

18. Siddiqui-Jain A, Grand CL, Bearss DJ, Hurley LH. Proc. Natl. Acad. Sci. U.S.A. 2002; 99:115938. [PubMed: 12195017]

19. Phan AT, Modi YS, Patel DJ. J. Am. Chem. Soc. 2004; 126:8710-6. [PubMed: 15250723]

20. Ambrus A, Chen D, Dai J, Jones RA, Yang D. Biochemistry. 2005; 44:2048-58. [PubMed: 15697230]

21. Hurley LH, Von Hoff DD, Siddiqui-Jain A, Yang D. Semin. Oncol. 2006; 33:498-512. [PubMed: 16890804]

22. Liu JN, Deng R, Guo JF, Zhou JM, Feng GK, Huang ZS, Gu LQ, Zeng YX, Zhu XF. Leukemia. 2007; 21:1300-2. [PubMed: 17392822]

23. Sun D, Hurley LH. J. Med. Chem. 2009; 52:2863-74. [PubMed: 19385599]

24. Yarden Y, Kuang WJ, Yang-Feng T, Coussens L, Munemitsu S, Dull TJ, Chen E, Schlessinger J, Francke U, Ullrich A. EMBO J. 1987; 6:3341-51. [PubMed: 2448137]

25. Roskoski R Jr. Biochem. Biophys. Res. Commun. 2005; 338:1307-15. [PubMed: 16226710]

26. Yang J, Du X, Lazar AJ, Pollock R, Hunt K, Chen K, Hao X, Trent J, Zhang W. Cancer. 2008; 113:1532-43. [PubMed: 18671247]

27. Hoeben A, Schoffski P, Debiec-Rychter M. Br. J. Cancer. 2008; 98:684-8. [PubMed: 18253129]

28. Tuveson DA, Willis NA, Jacks T, Griffin JD, Singer S, Fletcher CD, Fletcher JA, Demetri GD. Oncogene. 2001; 20:5054-8. [PubMed: 11526490]

29. Zhu MJ, Ou WB, Fletcher CD, Cohen PS, Demetri GD, Fletcher JA. Oncogene. 2007; 26:6386-95. [PubMed: 17452978]

30. Mol CD, Dougan DR, Schneider TR, Skene RJ, Kraus ML, Scheibe DN, Snell GP, Zou H, Sang BC, Wilson KP. J. Biol. Chem. 2004; 279:31655-63. [PubMed: 15123710]

31. Heinrich MC, Corless CL, Demetri GD, Blanke CD, von Mehren M, Joensuu H, McGreevey LS, Chen CJ, Van den Abbeele AD, Druker BJ, Kiese B, Eisenberg B, Roberts PJ, Singer S, Fletcher CD, Silberman S, Dimitrijevic S, Fletcher JA. J. Clin. Oncol. 2003; 21:4342-9. [PubMed: 14645423]

32. Heinrich MC, Corless CL, Blanke CD, Demetri GD, Joensuu H, Roberts PJ, Eisenberg BL, von Mehren M, Fletcher CD, Sandau K, McDougall K, Ou WB, Chen CJ, Fletcher JA. J. Clin. Oncol. 2006; 24:4764-74. [PubMed: 16954519]

33. von Mehren M. Clin. Colorectal Cancer. 2006; 6:S30-34. [PubMed: 17419150]

34. Le Tourneau C, Raymond E, Faivre S. Ther. Clin. Risk Manage. 2007; 3:341-8.

35. Liegl B, Kepten I, Le C, Zhu M, Demetri GD, Heinrich MC, Fletcher CD, Corless CL, Fletcher JA. J. Pathol. 2008; 216:64-74. [PubMed: 18623623]

36. Bejugam M, Sewitz S, Shirude PS, Rodriguez R, Shahid R, Balasubramanian S. J. Am. Chem. Soc. 2007; 129:12926-7. [PubMed: 17918848]

37. Gunaratnam M, Swank S, Haider SM, Galesa K, Reszka AP, Beltran M, Cuenca F, Fletcher JA, Neidle S. J. Med. Chem. 2009; 52:3774-83. [PubMed: 19469547]

38. Yamamoto K, Tojo A, Aoki N, Shibuya M. Jpn. J. Cancer Res. 1993; 84:1136-44. [PubMed: 7506248]

39. Rankin S, Reszka AP, Huppert J, Zloh M, Parkinson GN, Todd AK, Ladame S, Balasubramanian S, Neidle S. J. Am. Chem. Soc. 2005; 127:10584-9. [PubMed: 16045346]

40. Fernando H, Reszka AP, Huppert J, Ladame S, Rankin S, Venkitaraman AR, Neidle S, Balasubramanian S. Biochemistry. 2006; 45:7854-60. [PubMed: 16784237]

41. Phan AT, Kuryavyi V, Burge S, Neidle S, Patel DJ. J. Am. Chem. Soc. 2007; 129:4386-92. [PubMed: 17362008]

J Am Chem Soc. Author manuscript; available in PMC 2011 March 11. 
42. Todd AK, Haider SM, Parkinson GN, Neidle S. Nucleic Acids Res. 2007; 35:5799-808. [PubMed: 17720713]

43. Shirude PS, Okumus B, Ying L, Ha T, Balasubramanian S. J. Am. Chem. Soc. 2007; 129:7484-5. [PubMed: 17523641]

44. Phan AT, Patel DJ. J. Am. Chem. Soc. 2002; 124:1160-1. [PubMed: 11841271]

45. Schanda P, Kupce E, Brutscher B. J. Biomol. NMR. 2005; 33:199-211. [PubMed: 16341750]

46. Wüthrich, K. NMR of Protein and Nucleic Acids. John Wiley \& Sons, Inc.; New York: 1986.

47. Smith FW, Feigon J. Nature. 1992; 356:164-8. [PubMed: 1545871]

48. Krishna MMG, Hoang L, Lin Y, Englander SW. Methods. 2004; 34:51-64. [PubMed: 15283915]

49. Englander SW, Mayne L, Krishna MM. Q. Rev. Biophys. 2007; 40:287-326. [PubMed: 18405419]

50. Varnai P, Canalia M, Leroy JL. J. Am. Chem. Soc. 2004; 126:14659-67. [PubMed: 15521786]

51. Lee JH, Pardi A. Nucleic Acids Res. 2007; 35:2965-74. [PubMed: 17439958]

52. Every AE, Russu IM. Biopolymers. 2007; 87:165-73. [PubMed: 17636510]

53. Figueroa N, Keith G, Leroy JL, Plateau P, Roy S, Gueron M. Proc. Natl. Acad. Sci. U.S.A. 1983; 80:4330-3. [PubMed: 6348768]

54. Coman D, Russu IM. Nucleic Acids Res. 2004; 32:878-83. [PubMed: 14769945]

55. Phan AT, Kuryavyi V, Luu KN, Patel DJ. Nucleic Acids Res. 2007; 35:6517-25. [PubMed: 17895279]

56. Matsugami A, Xu Y, Noguchi Y, Sugiyama H, Katahira M. FEBS J. 2007; 274:3545-56. [PubMed: 17561958]

57. Phan AT, Patel DJ. J. Am. Chem. Soc. 2003; 125:15021-7. [PubMed: 14653736]

58. Zhang N, Phan AT, Patel DJ. J. Am. Chem. Soc. 2005; 127:17277-85. [PubMed: 16332077]

59. Phan AT, Kuryavyi V, Ma JB, Faure A, Andreola ML, Patel DJ. Proc. Natl. Acad. Sci. U.S.A. 2005; 102:634-9. [PubMed: 15637158]

60. Lane AN, Chaires JB, Gray RD, Trent JO. Nucleic Acids Res. 2008; 36:5482-515. [PubMed: 18718931]

61. Li J, Correia JJ, Wang L, Trent JO, Chaires JB. Nucleic Acids Res. 2005; 33:4649-59. [PubMed: 16106044]

62. Green JJ, Ying L, Klenerman D, Balasubramanian S. J. Am. Chem. Soc. 2003; 125:3763-7. [PubMed: 12656607]

63. Ying L, Green JJ, Li H, Klenerman D, Balasubramanian S. Proc. Natl. Acad. Sci. U.S.A. 2003; 100:14629-34. [PubMed: 14645716]

64. Lee JY, Okumus B, Kim DS, Ha T. Proc. Natl. Acad. Sci. U.S.A. 2005; 102:18938-43. [PubMed: 16365301]

65. Phan AT, Luu KN, Patel DJ. Nucleic Acids Res. 2006; 34:5715-9. [PubMed: 17040899]

66. Kaushik M, Bansal A, Saxena S, Kukreti S. Biochemistry. 2007; 46:7119-31. [PubMed: 17523598]

67. Dash J, Shirude PS, Hsu ST, Balasubramanian S. J. Am. Chem. Soc. 2008; 130:15950-6. [PubMed: 18980309]

68. Hazel P, Huppert J, Balasubramanian S, Neidle S. J. Am. Chem. Soc. 2004; 126:16405-15. [PubMed: 15600342]

69. Rachwal PA, Brown T, Fox KR. FEBS Lett. 2007; 581:1657-60. [PubMed: 17399710]

70. Dai J, Dexheimer TS, Chen D, Carver M, Ambrus A, Jones RA, Yang D. J. Am. Chem. Soc. 2006; 128:1096-8. [PubMed: 16433524]

71. Mergny JL, De Cian A, Amrane S, Webba da Silva M. Nucleic Acids Res. 2006; 34:2386-97. [PubMed: 16682446]

72. Spackova, N. a.; Berger, I.; Sponer, J. J. Am. Chem. Soc. 1999; 121:5519-5534.

73. Kouzine F, Sanford S, Elisha-Feil Z, Levens D. Nat. Struct. Mol. Biol. 2008; 15:146-54. [PubMed: 18193062]

74. Huppert JL. Philos. Trans. R. Soc., A. 2007; 365:2969-84.

75. Clarke J, Itzhaki LS, Fersht AR. Trends Biochem. Sci. 1997; 22:284-7. [PubMed: 9270297]

J Am Chem Soc. Author manuscript; available in PMC 2011 March 11. 
76. Campbell NH, Patel M, Tofa AB, Ghosh R, Parkinson GN, Neidle S. Biochemistry. 2009; 48:1675-80. [PubMed: 19173611]

77. Parkinson GN, Cuenca F, Neidle S. J. Mol. Biol. 2008; 381:1145-56. [PubMed: 18619463]

78. Cavanagh, J.; Fairbrother, WJ.; Palmer, AG., III; Rance, M.; Skelton, NJ. Protein NMR Spectroscopy. 2nd ed.. Academic Press; San Diego, CA: 2007.

79. Case, DA., et al. AMBER. 10 ed.. University of California; San Francisco, CA: 2008.

80. Wang JM, Cieplak P, Kollman PA. J. Comput. Chem. 2000; 21:1049-74.

81. Perez A, Marchan I, Svozil D, Sponer J, Cheatham TE 3rd. Laughton CA, Orozco M. Biophys. J. 2007; 92:3817-29. [PubMed: 17351000] 

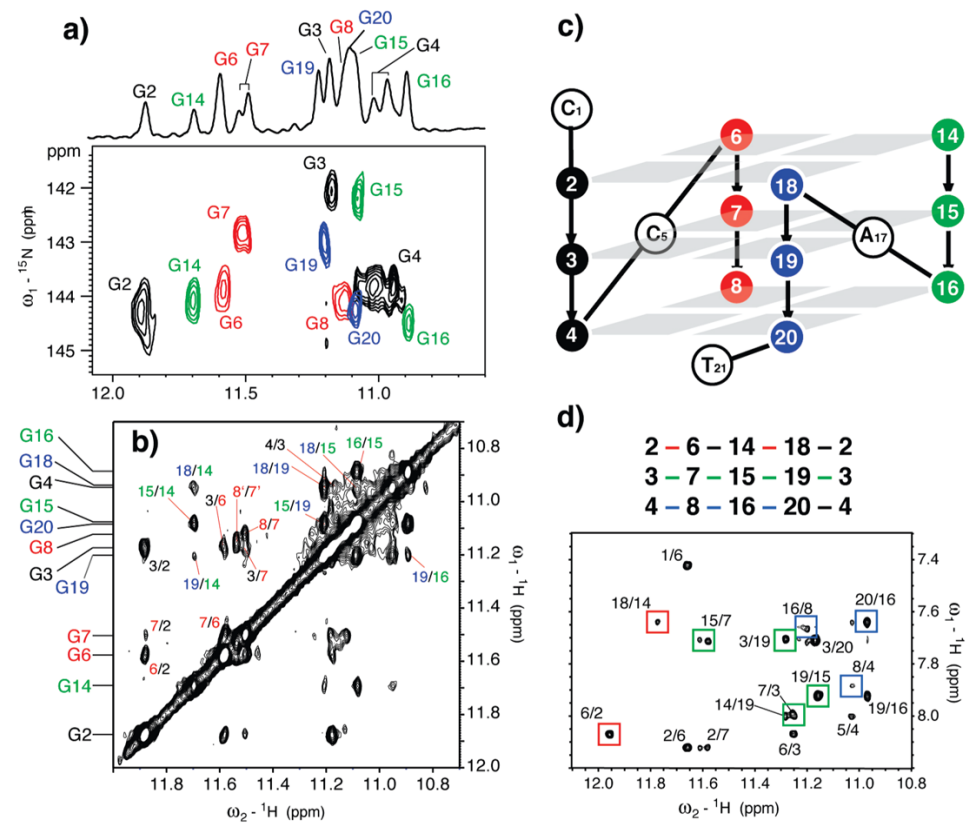

d)

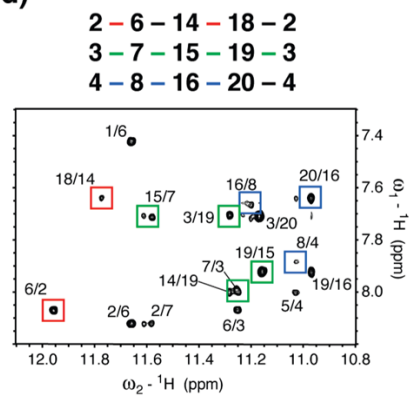

Figure 1.

Imino proton resonance assignments and G-quadruplex topology determination aided by site-specific ${ }^{15} \mathrm{~N}$ labeling. (a) Overlaid $2 \mathrm{D}{ }^{1} \mathrm{H}-{ }^{15} \mathrm{~N}$ correlation spectra of sitespecifically ${ }^{15} \mathrm{~N}$ labeled G21T oligonucleotides, recorded at $700 \mathrm{MHz}$ and at $298 \mathrm{~K}$.

Nucleotides that correspond to S1, S2, S3 and S4 are colored black, red, green and blue, respectively. The site-specific labeling enables unambiguous assignments of the 1D imino proton spectrum of G21T as shown on top of this figure. The presence of structural polymorphism is clearly visible for G4 and G7 in the 1D spectrum, and for G2 and G4 in the $2 \mathrm{D}$ spectrum. (b) Imino proton region of the $2 \mathrm{D}{ }^{1} \mathrm{H}$ NOESY spectrum of G21T. The assignments of the resolved cross-peaks are made through the imino proton resonance assignments obtained in panel (a) which are consistent with a G-quadruplex in a parallel conformation as shown in panel (c). (d) Interstrand NOEs between imino H1 (horizontal axis) and aromatic H8 (vertical axis) of unimolecular guanine bases within the same layer of G-tetrads (boxed in red, green and blue for the top, middle and bottom G-tetrads, respectively). Notably, G7H1 exhibits a minor conformation resulting in clear doubling. The expected connectivities between individual guanines are shown on top of this panel as dashes connecting the corresponding sequence numbers. Those that are observed in the NOESY spectrum are colored, accordingly. There are three cross-peaks (2/6, 2/7 and 14/19) that do not correspond to the unimolecular all-parallel topology and must arise due to the presence of an alternative conformation (see main text for details). The mixing time of this spectrum was $400 \mathrm{~ms}$ to yield better signals through spin-diffusion. 


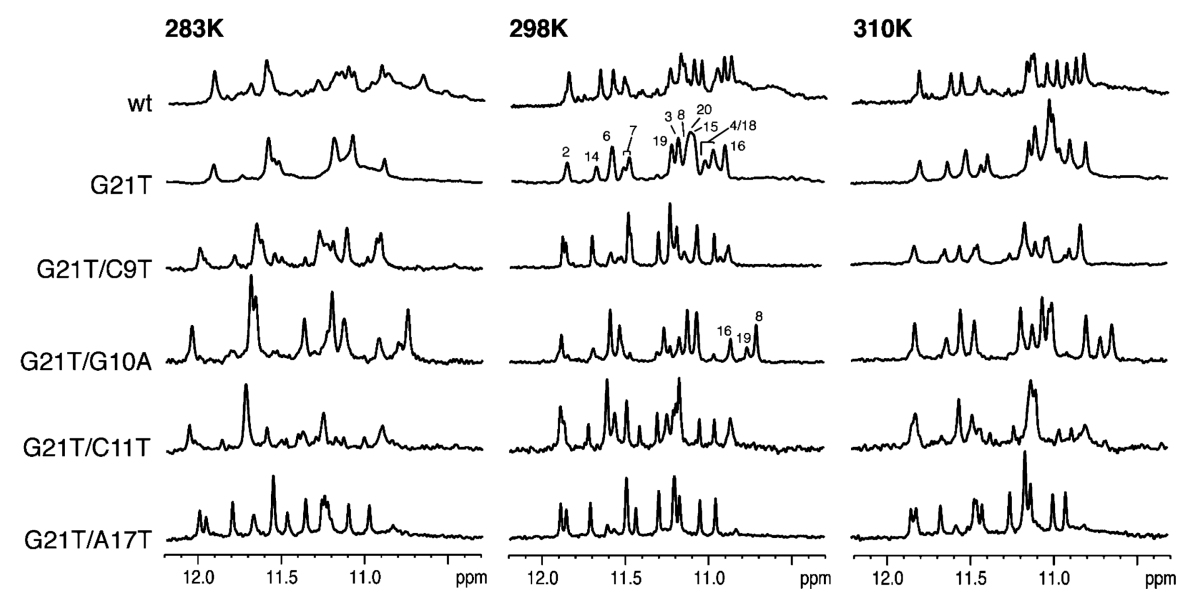

Figure 2.

The $700 \mathrm{MHz}$ imino proton spectra of c-kit2 variants recorded at different temperatures. The resonances in the spectrum of $\mathrm{G} 21 \mathrm{~T}$ recorded at $298 \mathrm{~K}$ are labeled with the corresponding sequence numbers to aid visualization. Additional assignments are made for G21T/G10A to highlight the upfield shifts of G8 H1 and G19 H1. Note that the spectrum of G21T/A17T at 298K, which does not change over time, resembles the initial spectrum of G21T (Figure S1). 

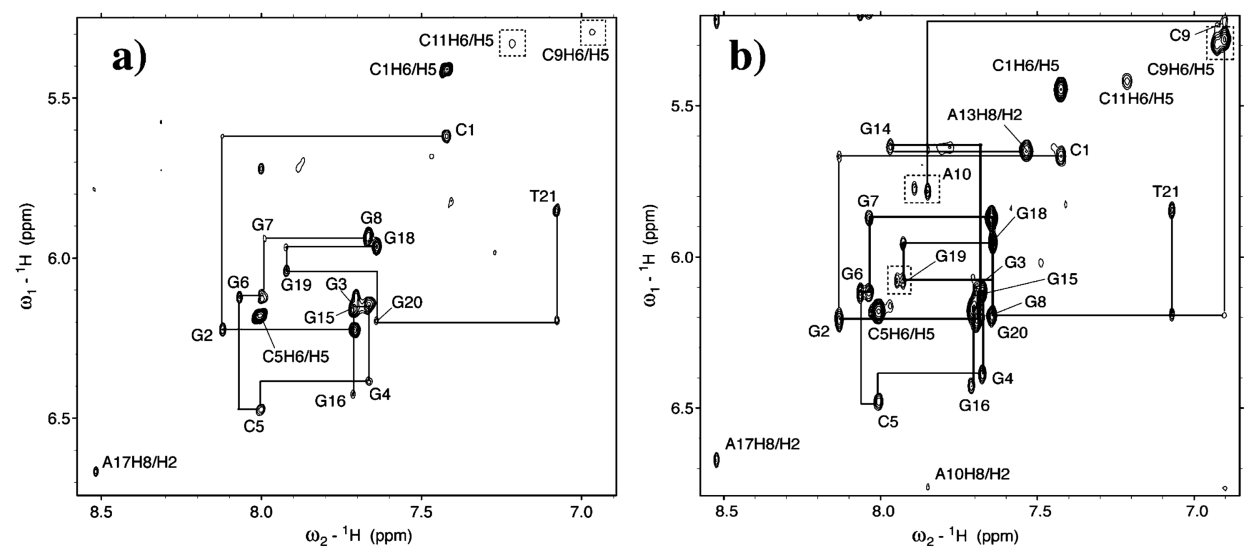

Figure 3.

NOE assignments and spectral comparisons of $c$-kit2 variants. Correlations between aromatic $\mathrm{H} 6 / \mathrm{H} 8$ and anomeric proton $\mathrm{H}^{\prime}{ }^{\prime}$ in the representative region of NOESY spectra of (a) G21T and (b) G21T/G10A were used to establish sequential connectivities between the spin systems. Intraresidue correlations are labeled with corresponding residue identities. For G21T, exceedingly weak intrabase H5/H6 correlations of C9 and C11 are indicated by dashed boxes to contrast with that of $\mathrm{C} 5$ and $\mathrm{C} 1$. The $\mathrm{G} 10 \mathrm{~A}$ replacement results in significant reduction in the dynamic range of observed peak intensities; at the same time, conformational polymorphism is clearly visible for a number of residues that give rise to two distinct cross-peaks (dashed boxes) with similar peak intensities. Both spectra were recorded at $700 \mathrm{MHz}$ and at $298 \mathrm{~K}$ with the same acquisition parameters and similar sample concentrations (ca. $200 \mu \mathrm{M}$ ). 
a)

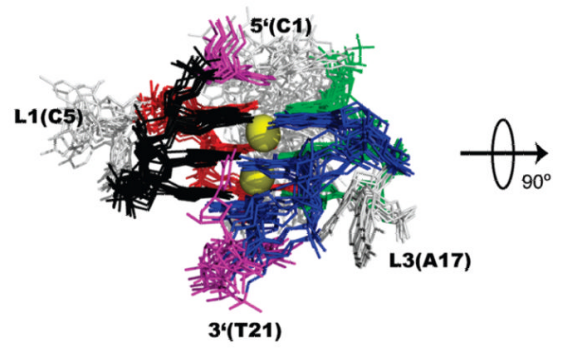

b)

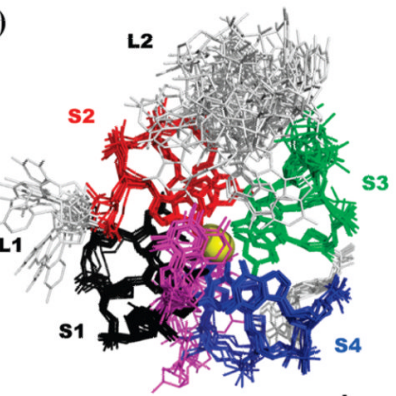

c)

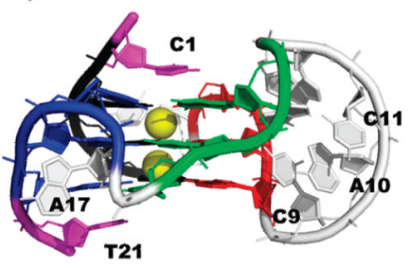

d)

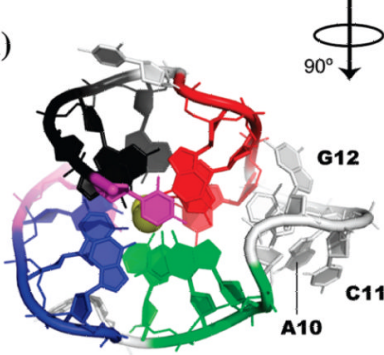

Figure 4.

Structural model of $c$-kit2 G21T/G10A. (a and b)Two orthogonal views of an ensemble of 10 lowest total energy structures of G21T/G10A. These structures have been subject to explicit water refinement with counterions included. Residues in S1, S2, S3 and S4 are colored in black, red, green and blue, respectively. S1 and S4 are capped by $\mathrm{C} 1$ and T21 at the $5^{\prime}$ and $3^{\prime}$ ends, respectively, shown in magenta. Two coordinated potassium ions, which have been included in the explicit solvent refinement procedure, are shown in yellow spheres. (c and d) Cartoon representation of the representative structural model. The structure is orthogonal from the ones in panels ( $a$ and $b$ ) to highlight the base stacking in L2, i.e., C9-C11, and the compaction of L3, i.e., A17, as labeled. 
a)

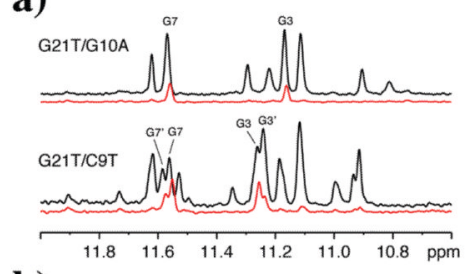

b)

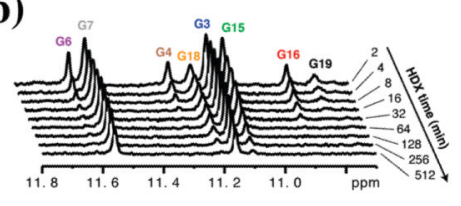

c)

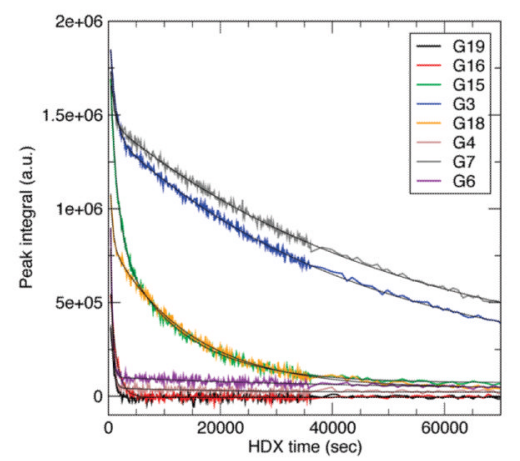

Figure 5.

NMR HDX kinetics monitored by real-time $1 \mathrm{D}{ }^{1} \mathrm{H}$ NMR spectroscopy. (a) The $900 \mathrm{MHz}$ 1D imino proton spectra of $c$-kit 2 variants recorded at time 0 and after $20 \mathrm{~h}$ of HDX at 298 $\mathrm{K}$. The experimental dead time is about 4 min during which time the resonances of a number of imino protons in the top G-tetrad have been lost, due to rapid exchange with bulk solvent. (b) Stack plot of a series of 1D imino proton spectra of G21T/G10A for following the kinetics of HDX in real-time. Each spectrum along the time series is taken from a geometric sequence, ranging from 2 to $512 \mathrm{~min}$. (c) Rate analysis of the c-kit2 G21T HDX kinetics. The NMR signals of each imino proton (colored as in panel (b)) are plotted as a function of time and fitted to a double-exponential function with a baseline offset. The fitted functions are shown in thin black lines. 

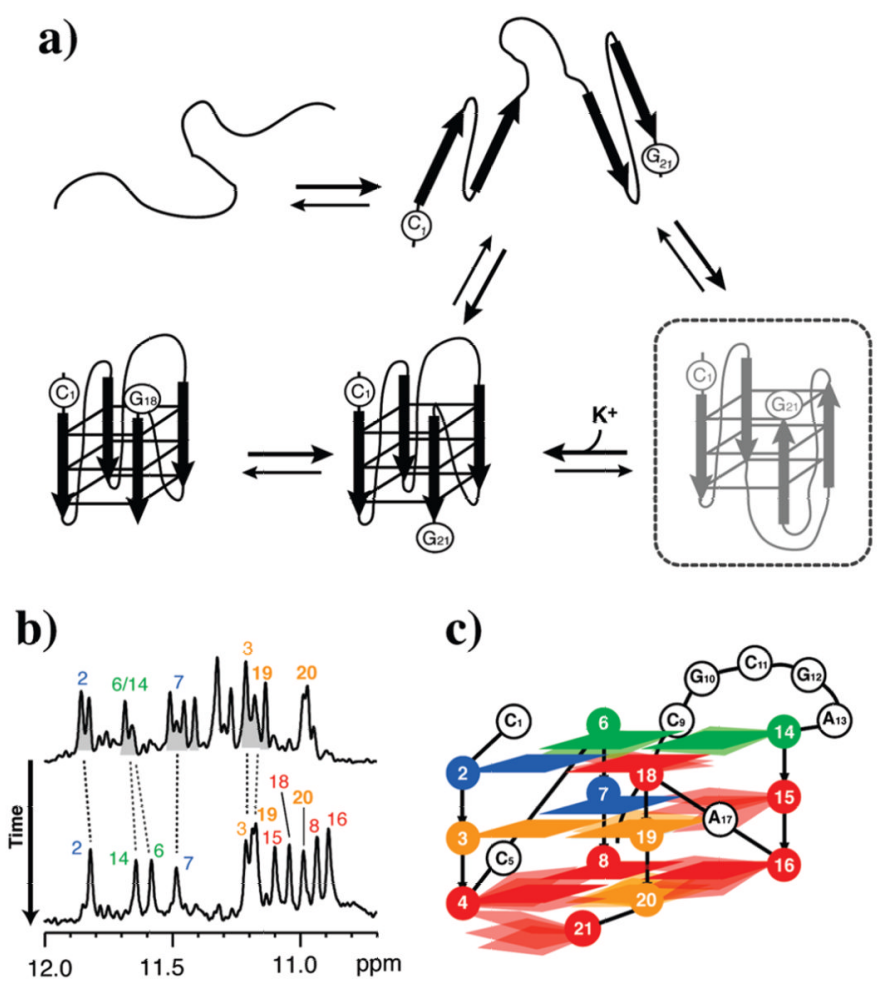

Figure 6.

Schematic representation of transitions between possible conformational arrangements of $c$ kit2. (a) The all parallel topology with two single nucleotide loops, L1 and L3, is the energetically most favorable conformation in the presence of potassium ions. Replacement of the last guanine in S4, G21T, reduces the complexity of the conformational space and therefore accelerates the process of conformational search for a low energy state in the associated free energy landscape. The gray dashed box highlights the presence of potassium ion-dependent conformation that is likely to be antiparallel (see Figure S3) whose detailed structure has hitherto not been characterized. (b) 1D imino proton spectra of wt c-kit2 recorded immediately after annealing (top panel) and after several months (bottom panel). Tentative assignments for the top spectrum are made based on chemical shift similarities with the ones that have been unambiguously assigned in the bottom spectrum with peaks that exhibit doublets (or more for G7 H1) shaded in gray. The resonances that have correspondence between the two spectra are connected by dashed line. The assignments of G2 and G7 are colored in blue for their respective chemical shift differences between the two spectra which are small; those of G6 and G16 are colored in green for they exhibit larger but well-resolved differences; those of G3, G19 and G20 are colored in orange for they have corresponding resonances in the initial spectrum (top panel) with very high ambiguities due to a large number of unresolved resonances for G4, G8, G15, G16, G18 and G21 (colored in red). (c) Schematic of the initial parallel topology attained by wt $c$-kit 2 with varied degrees of structural heterogeneities. The residues that have reached lower energy conformations, with different degrees of peak doublings, including G2 and G7 are shown in blue as in panel (b); ambiguous assignments were made for G3, G6, G14, G19 and G20 in panel (a) and they are shown in green and orange as in panel (b); the remaining residues, including G21 which may participate in G-tetrad formation in the early stage of the folding process in slightly different conformations, are shown in red with multiple parallelograms to indicate the structural heterogeneities. 


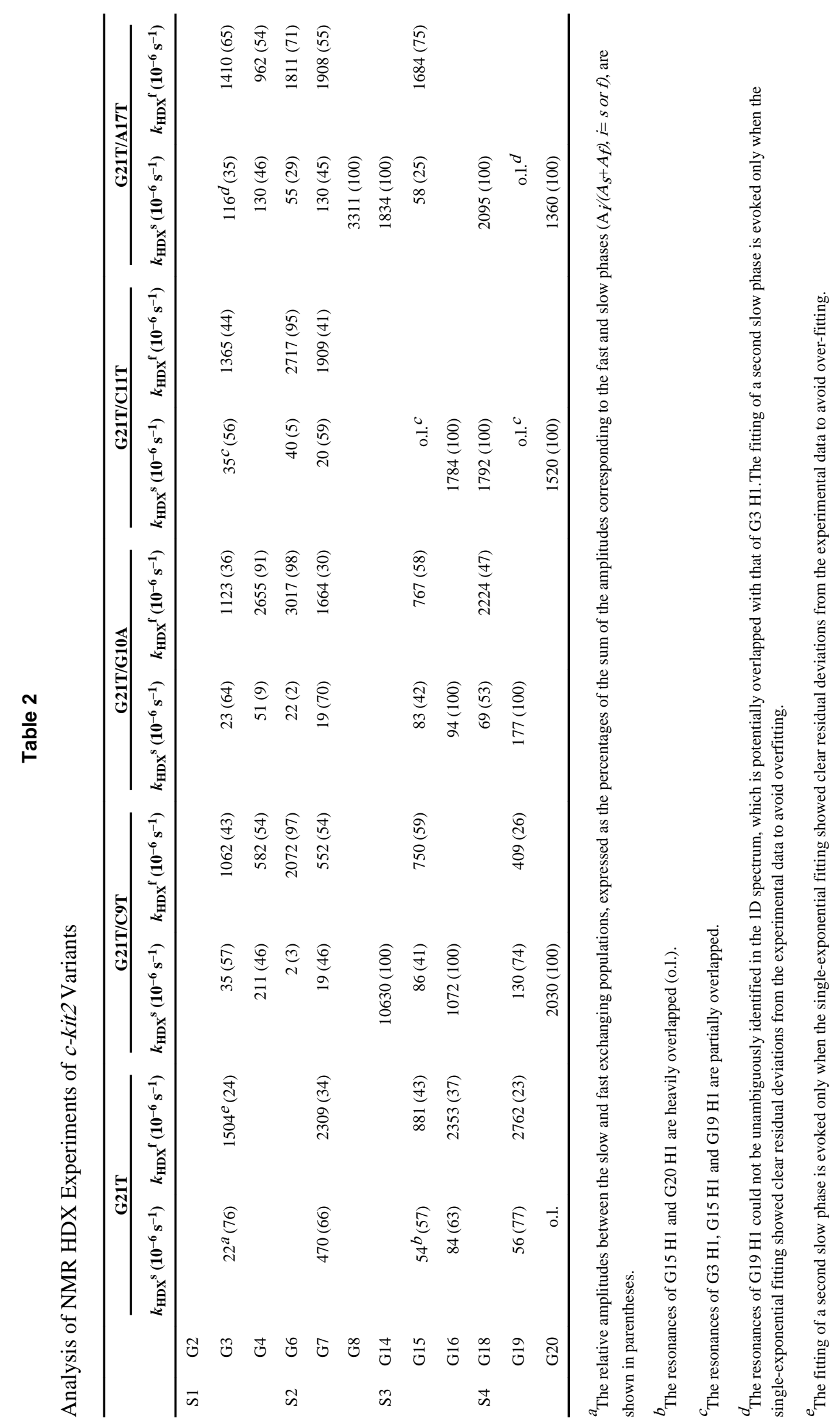

J Am Chem Soc. Author manuscript; available in PMC 2011 March 11. 